\title{
AN EFFECTIVE PROOF OF THE CARTAN FORMULA: THE EVEN PRIME
}

\author{
ANIBAL M. MEDINA-MARDONES
}

\begin{abstract}
The Cartan formula encodes the relationship between the cup product and the action of the Steenrod algebra in $\mathbb{F}_{p}$-cohomology. In this work, we present an effective proof of the Cartan formula at the cochain level when the field is $\mathbb{F}_{2}$. More explicitly, for an arbitrary pair of cocycles and any non-negative integer, we construct a natural coboundary that descends to the associated instance of the Cartan formula. Our construction works for general algebras over the Barratt-Eccles operad, in particular, for the singular cochains of spaces.
\end{abstract}

\section{INTRODUCTION}

Let $X$ be a space. In [Ste47, Steenrod introduced formulae to define his famous Steenrod squares

$$
S q^{k}: H^{*}\left(X ; \mathbb{F}_{2}\right) \rightarrow H^{*}\left(X ; \mathbb{F}_{2}\right)
$$

and in [SE62, he axiomatically characterized them by the following:

1. $S q^{k}$ is natural,

2. $S q^{0}$ is the identity,

3. $S q^{k}(x)=x^{2}$ for $x \in H^{-k}\left(X ; \mathbb{F}_{2}\right)$,

4. $S q^{k}(x)=0$ for $x \in H^{-n}\left(X ; \mathbb{F}_{2}\right)$ with $n>k$,

5. $S q^{k}(x y)=\sum_{i+j=k} S q^{i}(x) S q^{j}(y)$.

Axiom 5., known as the Cartan formula, is the focus of this work.

To describe our viewpoint and present the contributions of this paper in context, let us revisit some of the history of Steenrod's construction.

In the late thirties, Alexander, Whitney, and Čech defined the ring structure on cohomology

$$
[\alpha][\beta]=\left[\alpha \smile_{0} \beta\right]
$$

using a cochain level construction

$$
\smile_{0}: N^{*}(X ; \mathbb{Z}) \otimes N^{*}(X ; \mathbb{Z}) \rightarrow N^{*}(X ; \mathbb{Z})
$$

dual to a choice of simplicial chain approximation to the diagonal inclusion.

Steenrod then showed that $\smile_{0}$ is commutative up to coherent homotopies by effectively constructing cup- $i$ products

$$
\smile_{i}: N^{*}(X ; \mathbb{Z}) \otimes N^{*}(X ; \mathbb{Z}) \rightarrow N^{*}(X ; \mathbb{Z})
$$

enforcing its derived commutativity. (Axioms for these and connections with higher category theory can be found in MM18a and MM19]). Then, with coefficients in $\mathbb{F}_{2}$, Steenrod defined

$$
S q^{k}([\alpha])=\left[\alpha \smile_{k-n} \alpha\right] .
$$

This definition of the Steenrod squares makes the Cartan formula equivalent to

$$
0=\left[\left(\alpha \smile_{0} \beta\right) \smile_{i}\left(\alpha \smile_{0} \beta\right)+\sum_{i=j+k}\left(\alpha \smile_{j} \alpha\right) \smile_{0}\left(\beta \smile_{k} \beta\right)\right] .
$$


The goal of this work is to effectively construct for any $i \geq 0$ and arbitrary pair of cocycles $\alpha, \beta \in N^{*}\left(X ; \mathbb{F}_{2}\right)$ a natural cochain $\zeta_{i}(\alpha \otimes \beta)$ such that

$$
\delta \zeta_{i}(\alpha \otimes \beta)=\left(\alpha \smile_{0} \beta\right) \smile_{i}\left(\alpha \smile_{0} \beta\right)+\sum_{i=j+k}\left(\alpha \smile_{j} \alpha\right) \smile_{0}\left(\beta \smile_{k} \beta\right) .
$$

Following May70, we take a more general approach and in doing so we describe a non-necessarily effective construction for any $E_{\infty}$-algebra. We work over a fixed algebraic model $\mathcal{E}$ of the $E_{\infty}$-operad known as the Barratt-Eccles operad. This model, introduced by Berger-Fresse in BF04, is equipped with a diagonal map and the natural $\mathcal{E}$-algebra structure defined by these authors on the normalized cochains of simplicial sets is suitable for effective constructions.

This paper is part of an ongoing effort spearheaded by Greg Brumfiel and John Morgan to build effective models of classical homotopy-theoretic concepts, see for example [BM16] and [BM18. Motivation for this especific project came from a question of Anton Kapustin. See [KT17] for an instance where one of our formulae is used in the context of topological phases of matter.

Implementations of the constructions of this paper and of state-of-the-art algorithms for the computation of Steenrod squares and cup- $i$ products, as introduced in [MM18d], can be found in the author's website.

Acknowledgement. We would like to thank John Morgan, Greg Brumfiel, Dennis Sullivan, Anton Kapustin, Mark Behrens, Marc Stephan, and Kathryn Hess for their insights, questions, and comments about this project.

\section{Conventions AND PREliminaries}

For the remainder of this paper all algebraic constructions are considered over $\mathbb{F}_{2}$, the field with two elements.

2.1. Chain complexes and simplicial sets. We denote the category of chain complexes of $\mathbb{F}_{2}$-modules by Ch. Boundary maps decrease degree and for chain complexes $C$ and $C^{\prime}$ the set of linear maps $\operatorname{Hom}\left(C, C^{\prime}\right)$ is a chain complex with

$$
\operatorname{Hom}\left(C, C^{\prime}\right)_{n}=\left\{f: c \in C_{m} \text { implies } f(c) \in C_{m+n}^{\prime}\right\} \quad \partial f=\partial \circ f+f \circ \partial .
$$

Let $f, g, h \in \operatorname{Hom}\left(C, C^{\prime}\right)$. We remark that $f$ is a chain map if and only if it is a degree 0 cycle. Assume $f$ and $g$ are chain maps, then $h$ is a chain homotopy between them if and only if

$$
\partial h=f+g .
$$

We remark that $f \in \operatorname{Hom}\left(C, C^{\prime}\right)$ is a chain map if and only if it is a degree 0 cycle, and that $h \in \operatorname{Hom}\left(C, C^{\prime}\right)$ is a chain homotopy between two chain maps $f$ and $g$ if and only if

$$
\partial h=f+g .
$$

The product of two chain complexes $C$ and $C^{\prime}$ is defined by

$$
\left(C \otimes C^{\prime}\right)_{n}=\bigoplus_{i+j=n} C_{i} \otimes C_{j}^{\prime} \quad \partial=\partial \otimes \mathrm{id}+\mathrm{id} \otimes \partial .
$$

The category $\boldsymbol{\Delta}$ is defined to have an object $[n]=\{0, \ldots, n\}$ for every non-negative integer $n$ and a morphism $[m] \rightarrow[n]$ for each order-preserving function from $\{0, \ldots, m\}$ to $\{0, \ldots, n\}$.

For integers $0 \leq i \leq n$, the morphisms

$$
\delta_{i}:[n-1] \rightarrow[n] \quad \sigma_{i}:[n+1] \rightarrow[n]
$$

defined by

$$
\delta_{i}(k)=\left\{\begin{array}{ll}
k & k<i \\
k+1 & i \leq k
\end{array} \quad \text { and } \quad \sigma_{i}(k)= \begin{cases}k & k \leq i \\
k-1 & i<k\end{cases}\right.
$$


generate all morphisms in $\Delta$.

A simplicial set is a contravariant functor from $\boldsymbol{\Delta}$ to the category of sets. We denote the category of simplicial sets by sSet and use the standard notation

$$
X[n]=X_{n} \quad X\left(\delta_{i}\right)=d_{i} \quad X\left(\sigma_{i}\right)=s_{i} .
$$

The product of two simplicial sets $X$ and $Y$ is defined by

$$
(X \times Y)_{n}=X_{n} \times Y_{n} \quad d_{i}=d_{i} \times d_{i} \quad s_{i}=s_{i} \times s_{i} .
$$

The functor of normalized chains

$$
N_{*}: \text { sSet } \rightarrow \text { Ch }
$$

is defined by

$$
N_{n}(X)=\frac{\mathbb{F}_{2}\left\{X_{n}\right\}}{\mathbb{F}_{2}\left\{s\left(X_{n-1}\right)\right\}} \quad \partial_{n}=\sum_{i=0}^{n} d_{i}
$$

where $s\left(X_{n-1}\right)=\bigcup_{i=0}^{n-1} s_{i}\left(X_{n-1}\right)$.

The functor of normalized cochains $N^{*}$ is defined by composing $N_{*}$ with the linear duality functor $\operatorname{Hom}\left(-, \mathbb{F}_{2}\right): \mathbf{C h} \rightarrow \mathbf{C h}$. Notice that in this definition cochains are concentrated in non-positive degrees.

Remark 1. The singular cochains with $\mathbb{F}_{2}$-coefficients of a topological space $X$ coincide with $N^{*}(\operatorname{Sing}(X))$, where $\operatorname{Sing}(X)$ is the simplicial set of continuous maps from standard topological simplices to $X$.

2.2. The Alexander-Whitney and Eilenberg-Zilber maps. The functor of normalized chains does not preserve products, i.e., for a general pair of simplicial sets $X$ and $Y$ there is no isomorphism beetween $N_{*}(X \times Y)$ and $N_{*}(X) \otimes N_{*}(Y)$. Nevertheless, there is a canonical chain homotopy equivalence between them.

\section{The Alexander-Whitney map}

$$
A W: N_{*}(X \times Y) \rightarrow N_{*}(X) \otimes N_{*}(Y)
$$

is defined for $x \times y \in N_{n}(X \times Y)$ by

$$
A W(x \times y)=\sum_{i=0}^{n} d_{i+1} \cdots d_{n} x \otimes d_{0} \cdots d_{i-1} y
$$

and the Eilenberg-Zilber map

$$
E Z: N_{*}(X) \otimes N_{*}(Y) \rightarrow N_{*}(X \otimes Y)
$$

is defined for $x \otimes y \in N_{p}(X) \otimes N_{q}(Y)$ by

$$
E Z(x \otimes y)=\sum s_{v_{p}} \cdots s_{v_{1}} x \otimes s_{w_{q}} \cdots s_{w_{1}} y
$$

where the sum is over all pairs of disjoint subsets

$$
\left(\left\{v_{1}<\cdots<v_{p}\right\},\left\{w_{1}<\cdots<w_{q}\right\}\right)
$$

of $\{0, \ldots, p+q-1\}$.

It is well known that the compositions $A W E Z$ is equal to the identity and that the composition $E Z A W$ is effectively chain homotopic to the identity. A recursive description for one such chain homotopy was first given in [EML53]. A close formula for it, which we learned from Rea00] and is credited therein to Rub91, is given next.

The Shih homotopy

$$
S H I: N_{n}(X \times Y) \rightarrow N_{*}(X \times Y)
$$


is defined for $x \times y \in N_{n}(X \otimes Y)$ to be 0 if $n=0$ and if $n>0$ by

$$
S H I(x \times y)=\sum \begin{aligned}
& s_{v_{p}+m} \cdots s_{v_{1}+m} s_{m-1} d_{n-p+1} \cdots d_{n} x \otimes \\
& s_{w_{q+1}+m} \cdots s_{w_{1}+m} d_{n-p-q} \cdots d_{n-p-1} y
\end{aligned}
$$

where $m=n-p-q$ and the sum is over all pairs of disjoint subsets

$$
\left(\left\{v_{1}<\cdots<v_{p}\right\},\left\{w_{1}<\cdots<w_{q+1}\right\}\right)
$$

of $\{0, \ldots, p+q\}$ with $0 \leq p \leq n-1$ and $0 \leq q \leq n-p-1$.

2.3. Group actions and algebras over operads. Let $\Gamma$ be a group. A $\Gamma$-action on an object $C$ is a group morphism

$$
\Gamma \rightarrow \operatorname{Aut}(C)
$$

where the group structure on $\operatorname{Aut}(C)$ is given by composition.

Let $\mathcal{O}$ be an operad. (See for example [LV12.) An $\mathcal{O}$-algebra structure on an object $C$ is an operad morphism

$$
\mathcal{O} \rightarrow \operatorname{End}(C)
$$

where the operad structure on $\operatorname{End}(C)=\left\{\operatorname{Hom}\left(C^{\otimes n}, C\right)\right\}_{n \geq 0}$ is induced from composition of linear maps and transpositions of factors.

Let $C$ be an object with a $\Gamma$-action or an $\mathcal{O}$-algebra structure. We identify the elements of $\Gamma$ or $\mathcal{O}$ with their images via (5) or (6).

2.4. The Barratt-Eccles operad $\mathcal{E}$. We review from [BF04] the Barratt-Eccles operad.

For a positive integer $r$ let $\Sigma_{r}$ be the group of permutations of $r$ elements and

$$
\mathrm{o}_{\Sigma}: \Sigma_{r} \times \Sigma_{s_{1}} \times \cdots \times \Sigma_{s_{r}} \rightarrow \Sigma_{s_{1}+\cdots+s_{r}}
$$

the usual composition of permutations.

For a positive integer $r$ define the simplicial set $E(r)$ by

$$
\begin{aligned}
E(r)_{n} & =\left\{\left(\sigma_{0}, \ldots, \sigma_{n}\right) \mid \sigma_{i} \in \Sigma_{r}\right\}, \\
d_{i}\left(\sigma_{0}, \ldots, \sigma_{n}\right) & =\left(\sigma_{0}, \ldots, \widehat{\sigma}_{i}, \ldots, \sigma_{n}\right), \\
s_{i}\left(\sigma_{0}, \ldots, \sigma_{n}\right) & =\left(\sigma_{0}, \ldots, \sigma_{i}, \sigma_{i}, \ldots, \sigma_{n}\right) .
\end{aligned}
$$

We consider $E(r)$ equipped with the action of $\Sigma_{r}$ given by

$$
\sigma\left(\left(\sigma_{0}, \ldots, \sigma_{n}\right)\right)=\left(\sigma \sigma_{0}, \ldots, \sigma \sigma_{n}\right) .
$$

Let

$$
\circ_{E}: E(r) \times E\left(s_{1}\right) \times \cdots \times E\left(s_{r}\right) \rightarrow E\left(s_{1}+\cdots+s_{r}\right)
$$

be defined by applying $o_{\Sigma}$ coordinatewise.

For $r \geq 0$, let

$$
\mathcal{E}(r)=N_{*}(E(r))
$$

be edowed with the induced $\Sigma_{r}$-action. Define an operadic compositions $\circ_{\mathcal{E}}$ on $\mathcal{E}=\{\mathcal{E}(r)\}_{r \geq 0}$ by

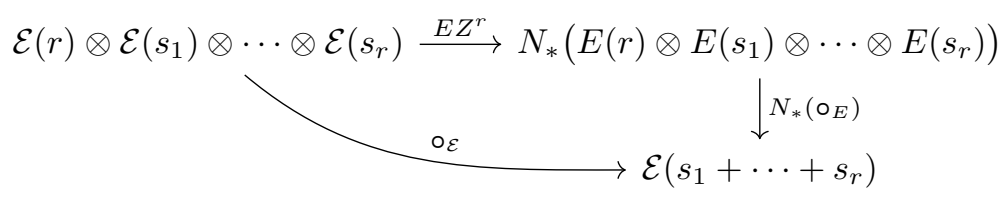


where $E Z^{r}$ stand recursively for $E Z\left(\mathrm{id} \otimes E Z^{r-1}\right)$ with $E Z^{1}=E Z$.

The resulting operad $\mathcal{E}$ is referred to as the Barratt-Eccles operad. It is a model in the category $\mathbf{C h}$ for the $E_{\infty}$-operad. That is to say, $E(0)=\mathbb{F}_{2}$ and, for $r>0, E(r)$ is a resolution of $\mathbb{F}_{2}$ by free $\mathbb{F}_{2}\left[\Sigma_{r}\right]$-modules.

\section{Cartan coboundaries for $\mathcal{E}$-Algebras}

3.1. Steenrod cup- $i$ products and Cartan coboundaries. Since $\mathcal{E}$ is an $E_{\infty}$-operad, the orbit complex $\mathcal{E}(2)_{\Sigma_{2}}$ is an algebraic model for $K\left(\mathbb{F}_{2}, 1\right)$, and $H_{i}\left(\mathcal{E}(2)_{\Sigma_{2}}\right)$ is generated as an $\mathbb{F}_{2}$-module by the orbit of the element

$$
\tilde{x}_{i}=\left(e,(12), e, \ldots,(12)^{i}\right) .
$$

Definition 2. Let $A$ be an $\mathcal{E}$-algebra. The cup- $i$ product of $A$ is the image of $\tilde{x}_{i}$ in $\operatorname{Hom}(A \otimes A, A)$. We use the notation

$$
\tilde{x}_{i}(\alpha \otimes \beta)=\alpha \smile_{i} \beta .
$$

A Cartan $i$-coboundary is any map $\zeta_{i}$ in $\operatorname{Hom}(A \otimes A, A)$ that satisfies

$$
\left(\partial \zeta_{i}\right)(\alpha \otimes \beta)=\left(\alpha \smile_{0} \beta\right) \smile_{i}\left(\alpha \smile_{0} \beta\right)+\sum_{i=j+k}\left(\alpha \smile_{j} \alpha\right) \smile_{0}\left(\beta \smile_{k} \beta\right) .
$$

Definition 3. The chain maps $F, G: \mathcal{E}(2) \rightarrow \mathcal{E}(4)$ are defined by

$$
\begin{aligned}
& F\left(\sigma_{0}, \ldots, \sigma_{n}\right)=\circ_{\mathcal{E}}\left(\left(\sigma_{0}, \ldots, \sigma_{n}\right) \otimes \tilde{x}_{0} \otimes \tilde{x}_{0}\right) \\
& G\left(\sigma_{0}, \ldots, \sigma_{n}\right)=\circ_{\mathcal{E}}\left(\tilde{x}_{0} \otimes A W\left(\sigma_{0}, \ldots, \sigma_{n}\right)^{\otimes 2}\right)
\end{aligned}
$$

for any basis element $\left(\sigma_{0}, \ldots, \sigma_{n}\right)$.

Lemma 4. Let $A$ be an $\mathcal{E}$-algebra. For $\alpha, \beta \in A$ and $i \geq 0$ we have

$$
(23) F\left(\tilde{x}_{i}\right)(\alpha \otimes \alpha \otimes \beta \otimes \beta)=\left(\alpha \smile_{0} \beta\right) \smile_{i}\left(\alpha \smile_{0} \beta\right)
$$

and

$$
G\left(\tilde{x}_{i}\right)(\alpha \otimes \alpha \otimes \beta \otimes \beta)=\sum_{i=j+k}\left(\alpha \smile_{j} \alpha\right) \smile_{0}\left(\beta \smile_{k} \beta\right) .
$$

Proof. For (7) we compute

$$
\begin{aligned}
(23) F\left(\tilde{x}_{i}\right)(\alpha \otimes \beta \otimes \alpha \otimes \beta) & =(23) \circ_{\mathcal{E}}\left(\tilde{x}_{i} \otimes \tilde{x}_{0} \otimes \tilde{x}_{0}\right)(\alpha \otimes \alpha \otimes \beta \otimes \beta) \\
& =\circ_{\mathcal{E}}\left(\tilde{x}_{i} \otimes \tilde{x}_{0} \otimes \tilde{x}_{0}\right)(\alpha \otimes \beta \otimes \alpha \otimes \beta) \\
& =\tilde{x}_{i}\left(\tilde{x}_{0}(\alpha \otimes \beta) \otimes \tilde{x}_{0}(\alpha \otimes \beta)\right) \\
& =\left(\alpha \smile_{0} \beta\right) \smile_{i}\left(\alpha \smile_{0} \beta\right) .
\end{aligned}
$$

For (8) we notice that

$$
A W\left(\tilde{x}_{i} \otimes \tilde{x}_{i}\right)=\sum_{j=0}^{i} \tilde{x}_{j} \otimes(12)^{j} \tilde{x}_{i-j}
$$

and compute

$$
\begin{aligned}
G\left(\tilde{x}_{i}\right)(\alpha \otimes \alpha \otimes \beta \otimes \beta) & =\sum_{j=0}^{i} \circ_{\mathcal{E}}\left(\tilde{x}_{0} \otimes \tilde{x}_{j} \otimes(12)^{j} \tilde{x}_{i-j}\right)(\alpha \otimes \alpha \otimes \beta \otimes \beta) \\
& =\sum_{j=0}^{i} \tilde{x}_{0}\left(\tilde{x}_{j}(\alpha \otimes \alpha) \otimes \tilde{x}_{i-j}(\beta \otimes \beta)\right) \\
& =\sum_{i=j+k}\left(\alpha \smile_{j} \alpha\right) \smile_{0}\left(\beta \smile_{k} \beta\right)
\end{aligned}
$$

as desired. 
Lemma 5. Let $H: \mathcal{E}(2) \rightarrow \mathcal{E}(4)$ be a chain homotopy between $(23) \circ F$ and $G$ satisfying

$$
H \circ(12)=(12)(34) \circ H .
$$

Then, for any $\mathcal{E}$-algebra $A$ and $i \geq 0$, the map $\zeta_{i}: A \otimes A \rightarrow A$ defined by

$$
\zeta_{i}(\alpha \otimes \beta)=H\left(\tilde{x}_{i}\right)(\alpha \otimes \alpha \otimes \beta \otimes \beta)
$$

is a Cartan i-coboundary.

Proof. By assumption

$$
\partial H=\partial \circ H+H \circ \partial=(23) \circ F+G
$$

so evaluating on $\tilde{x}_{i}$ we get

$$
\partial\left(H\left(\tilde{x}_{i}\right)\right)+H\left((12) \tilde{x}_{i-1}+\tilde{x}_{i-1}\right)=(23) F\left(\tilde{x}_{i}\right)+G\left(\tilde{x}_{i}\right) .
$$

Since $((12)(34)+\mathrm{id})(\alpha \otimes \alpha \otimes \beta \otimes \beta)=0$, the equivariance assumption implies

$$
H\left((12) \tilde{x}_{i-1}+\tilde{x}_{i-1}\right)(\alpha \otimes \alpha \otimes \beta \otimes \beta)=0 .
$$

Evaluating (9) on $(\alpha \otimes \alpha \otimes \beta \otimes \beta)$ and using Lemma 4 we obtain

$$
\left(\partial \zeta_{i}\right)(\alpha \otimes \beta)=\left(\alpha \smile_{0} \beta\right) \smile_{i}\left(\alpha \smile_{0} \beta\right)+\sum_{i=j+k}\left(\alpha \smile_{j} \alpha\right) \smile_{0}\left(\beta \smile_{k} \beta\right)
$$

as desired.

3.2. Statement of the main theorem. We now describe the construction of a chain homotopy as stated in Lemma 5 .

The first step of our construction is motivated by the well known fact that two group homomorphisms that are conjugate of each other induce homotopic maps of classifying spaces.

Let us consider the three group inclusions

$$
i_{1}(\sigma)=\sigma \times e \quad i_{2}(\sigma)=e \times \sigma \quad D(\sigma)=\sigma \times \sigma .
$$

of $\Sigma_{2}$ into $\Sigma_{2} \times \Sigma_{2}$.

Definition 6. The homomorphisms $f, g: \Sigma_{2} \rightarrow \Sigma_{4}$ are defined by

$$
\begin{aligned}
& f=o_{\Sigma} \circ(\mathrm{id} \times D) \circ i_{1} \\
& g=o_{\Sigma} \circ(\mathrm{id} \times D) \circ i_{2}
\end{aligned}
$$

or, more explicitly, by

$$
\begin{array}{ll}
f(e)=e & f(12)=(13)(24) \\
g(e)=e & g(12)=(12)(34) .
\end{array}
$$

We notice that the maps $f$ and $g$ are conjugated by (23) in $\Sigma_{4}$, i.e., for $\sigma \in \Sigma_{2}$

$$
(23) f(\sigma)=g(\sigma)(23) \text {. }
$$

Definition 7. Let $F, G: E(2) \rightarrow E(4)$ be the simplicial maps induced from $f$ and $g$ respectively. Explicitly,

$$
\begin{aligned}
& E(f)\left(\sigma_{0}, \ldots, \sigma_{n}\right)=\left(f \sigma_{0}, \ldots, f \sigma_{n}\right) \\
& E(g)\left(\sigma_{0}, \ldots, \sigma_{n}\right)=\left(g \sigma_{0}, \ldots, g \sigma_{n}\right)
\end{aligned}
$$

for any $\left(\sigma_{0}, \ldots, \sigma_{n}\right) \in E(2)$.

We introduce a linear map $H_{1}$ which, as we will see later, is an appropriately equivariant chain homotopy between $N_{*}(E(f))$ and $N_{*}(E(g))$. 
Definition 8. Let $H_{1}: \mathcal{E}(2) \rightarrow \mathcal{E}(4)$ be the degree 1 linear map defined on basis elements by

$$
H_{1}\left(\sigma_{0}, \ldots, \sigma_{n}\right)=\sum_{i=0}^{n}\left((23) f \sigma_{0}, \ldots,(23) f \sigma_{i}, g \sigma_{i}, \ldots, g \sigma_{n}\right) \text {. }
$$

The second step relates the maps $F$ and $N_{*}(E(f))$, as well as $G$ and $N_{*}(E(g))$. It turns out, as we will see later, that the maps in the first pair are equal, whereas the maps in the second are chain homotopic via an appropriately equivariant map that we now introduce.

Definition 9. Let $H_{2}: \mathcal{E}(2) \rightarrow \mathcal{E}(4)$ be the degree 1 linear map defined on basis elements by

$$
H_{2}\left(\sigma_{0}, \ldots, \sigma_{n}\right)=N_{*}\left(o_{E}\right)\left((e, \ldots, e) \otimes S H I\left(\sigma_{0}, \ldots, \sigma_{n}\right)^{\otimes 2}\right) \text {. }
$$

We are ready to state our main result.

Theorem 10. Let $A$ be an $\mathcal{E}$-algebra. For any $i \geq 0$ the map $\zeta_{i}$ in $\operatorname{Hom}(A \otimes A, A)$ defined by

$$
\zeta_{i}(\alpha \otimes \beta)=\left(H_{1}+H_{2}\right)\left(\tilde{x}_{i}\right)(\alpha \otimes \alpha \otimes \beta \otimes \beta)
$$

is a Cartan i-coboundary.

3.3. Proof of the main theorem. We will prove Theorem 10 after four lemmas.

Lemma 11. In $\operatorname{Hom}(\mathcal{E}(2), \mathcal{E}(4))$

$$
\partial H_{1}=(23) \circ N_{*}(E(f))+N_{*}(E(g)) .
$$

Proof. The essence of the proof is a telescopic sum argument.

$$
\begin{aligned}
H_{1} \partial\left(\sigma_{0}, \ldots, \sigma_{n}\right) & =H_{1} \sum_{j=0}^{n}\left(\sigma_{0}, \ldots, \widehat{\sigma}_{j}, \ldots, \sigma_{n}\right) \\
& =\sum_{j=0}^{n} \sum_{i<j}\left((23) f \sigma_{0}, \ldots,(23) f \sigma_{i}, g \sigma_{i}, \ldots, \widehat{\sigma}_{j}, \ldots, g \sigma_{n}\right) \\
& +\sum_{j=0}^{n} \sum_{i>j}\left((23) f \sigma_{0}, \ldots, \widehat{\sigma}_{j}, \ldots(23) f \sigma_{i}, g \sigma_{i}, \ldots, g \sigma_{n}\right) \\
& =\partial H_{1}\left(\sigma_{0}, \ldots, \sigma_{n}\right) \\
& +\sum_{i}\left(d_{i}+d_{i+1}\right)\left((23) f \sigma_{0}, \ldots,(23) f \sigma_{i}, g \sigma_{i}, \ldots, g \sigma_{n}\right) \\
& =\partial H_{1}\left(\sigma_{0}, \ldots, \sigma_{n}\right) \\
& +(23) N_{*}(E(f))\left(\sigma_{0}, \ldots, \sigma_{n}\right)+N_{*}(E(g))\left(\sigma_{0}, \ldots, \sigma_{n}\right)
\end{aligned}
$$

for any basis element $\left(\sigma_{0}, \ldots, \sigma_{n}\right) \in \mathcal{E}(2)$.

Lemma 12. The map $H_{1}$ satisfies the following form of equivariance:

$$
H_{1} \circ(12)=(12)(34) \circ H_{1} \text {. }
$$

Proof. Let $H_{1}^{i}$ be the degree 1 linear map defined on basis elements by

$$
H_{1}^{i}\left(\sigma_{0}, \ldots, \sigma_{n}\right)=\left((23) f \sigma_{0}, \ldots,(23) f \sigma_{i}, g \sigma_{i}, \ldots, g \sigma_{n}\right) .
$$

Notice that $H_{1}=\sum_{i} H_{1}^{i}$. We will verify (12) by checking it for each $H_{1}^{i}$. Using (10) we notice that for any $\sigma$ in $\Sigma_{2}$ we have

$$
\begin{aligned}
(23) f \sigma & =(g \sigma)(23) \\
g((12) \sigma) & =(12)(34) g \sigma .
\end{aligned}
$$


Therefore,

$$
\begin{aligned}
(12)(34) & H_{1}^{i}\left(\sigma_{0}, \ldots, \sigma_{n}\right) \\
& =(12)(34)\left((23) f \sigma_{0}, \ldots,(23) f \sigma_{i}, g \sigma_{i}, \ldots, g \sigma_{n}\right) \\
& =(12)(34)\left(g \sigma_{0}(23), \ldots, g \sigma_{i}(23), g \sigma_{i}, \ldots, g \sigma_{n}\right) \\
& =\left(g\left((12) \sigma_{0}\right)(23), \ldots, g\left((12) \sigma_{i}\right)(23), g\left((12) \sigma_{i}\right), \ldots, g\left((12) \sigma_{n}\right)\right) \\
& =\left((23) f\left((12) \sigma_{0}\right), \ldots,(23) f\left((12) \sigma_{i}\right), g\left((12) \sigma_{i}\right), \ldots, g\left((12) \sigma_{n}\right)\right) \\
& =H_{1}^{i}(12)\left(\sigma_{0}, \ldots, \sigma_{n}\right)
\end{aligned}
$$

for any basis element $\left(\sigma_{0}, \ldots, \sigma_{n}\right) \in \mathcal{E}(2)$.

Lemma 13. In $\operatorname{Hom}(\mathcal{E}(2), \mathcal{E}(4))$

$$
N_{*}(E(f))=F
$$

and

$$
\partial H_{2}=N_{*}(E(g))+G .
$$

Proof. We have

$$
\begin{aligned}
F\left(\sigma_{0}, \ldots, \sigma_{n}\right) & =\circ_{\mathcal{E}}\left(\left(\sigma_{0}, \ldots, \sigma_{n}\right) \otimes \tilde{x}_{0} \otimes \tilde{x}_{0}\right) \\
& =N_{*}\left(\circ_{E}\right)\left(\left(\sigma_{0}, \ldots, \sigma_{n}\right) \otimes(e, \ldots, e) \otimes(e, \ldots, e)\right) \\
& =\left(f \sigma_{0}, \ldots, f \sigma_{n}\right)=N_{*}(E(f))\left(\sigma_{0}, \ldots, \sigma_{n}\right)
\end{aligned}
$$

and

$$
\begin{aligned}
G\left(\sigma_{0}, \ldots, \sigma_{n}\right) & =o_{\mathcal{E}}\left(\tilde{x}_{0} \otimes A W\left(\sigma_{0}, \ldots, \sigma_{n}\right)^{\otimes 2}\right) \\
& =N_{*}\left(\circ_{E}\right)\left((e, \ldots, e) \otimes E Z A W\left(\sigma_{0}, \ldots, \sigma_{n}\right)^{\otimes 2}\right) \\
& =N_{*}\left(\circ_{E}\right)\left((e, \ldots, e) \otimes\left(\sigma_{0}, \ldots, \sigma_{n}\right)^{\otimes 2}\right) \\
& +N_{*}\left(o_{E}\right)\left((e, \ldots, e) \otimes(\partial S H I)\left(\sigma_{0}, \ldots, \sigma_{n}\right)^{\otimes 2}\right) \\
& =\left(g \sigma_{0}, \ldots, g \sigma_{n}\right)+\left(\partial H_{2}\right)\left(\sigma_{0}, \ldots, \sigma_{n}\right) \\
& =N_{*}(E(g))\left(\sigma_{0}, \ldots, \sigma_{n}\right)+\left(\partial H_{2}\right)\left(\sigma_{0}, \ldots, \sigma_{n}\right)
\end{aligned}
$$

for any basis element $\left(\sigma_{0}, \ldots, \sigma_{n}\right) \in \mathcal{E}(2)$.

Lemma 14. The map $\mathrm{H}_{2}$ satisfies the following form of equivariance:

$$
H_{2} \circ(12)=(12)(34) \circ H_{2} .
$$

Proof. Since for any $\sigma, \sigma^{\prime} \in \Sigma_{2}$

$$
\circ_{\boldsymbol{\Sigma}}\left(e,(12) \sigma,(12) \sigma^{\prime}\right)=(12)(34) \circ_{\boldsymbol{\Sigma}}\left(e, \sigma, \sigma^{\prime}\right)
$$

we have

$$
\begin{aligned}
H_{2}(12)\left(\sigma_{0}, \ldots, \sigma_{n}\right) & =N_{*}\left(\mathrm{o}_{E}\right)\left((e, \ldots, e) \otimes S H I\left((12) \sigma_{0}, \ldots,(12) \sigma_{n}\right)^{\otimes 2}\right) \\
& =N_{*}\left(\mathrm{o}_{E}\right)\left((e, \ldots, e) \otimes((12) \otimes(12)) S H I\left(\sigma_{0}, \ldots, \sigma_{n}\right)^{\otimes 2}\right) \\
& =(12)(34) N_{*}\left(\mathrm{o}_{E}\right)\left((e, \ldots, e) \otimes S H I\left(\sigma_{0}, \ldots, \sigma_{n}\right)^{\otimes 2}\right) \\
& =(12)(34) H_{2}\left(\sigma_{0}, \ldots, \sigma_{n}\right)
\end{aligned}
$$

for any basis element $\left(\sigma_{0}, \ldots, \sigma_{n}\right) \in \mathcal{E}(2)$. 
Proof of Theorem 10. We will show that $H=H_{1}+H_{2}$ satisfies the hypothesis of Lemma 5 Notice that the equivariance condition follows from Lemma 12 and Lemma 14. To verify that $H$ is a chain homotopy between $(23) \circ F$ and $G$ we use Lemma 11 and Lemma 13

$$
\begin{aligned}
\partial H & =\partial H_{1}+\partial H_{2} \\
& =(23) \circ N_{*}(E(f))+N_{*}(E(g))+N_{*}(E(g))+G \\
& =(23) \circ F+G
\end{aligned}
$$

as desired.

\section{The $\mathcal{E}$-ALGEBRa STRUCTURE ON NORMALIZED COCHAins}

In this section we effectively describe an $\mathcal{E}$-algebra structure on the normalized cochains of simplicial sets. This will allow us to apply our construction of Cartan $i$-coboundaries to this central example. Following [BF04, we achive this in two steps corresponding to the two operad morphisms

$$
\mathcal{E} \rightarrow \operatorname{Sur} \rightarrow \operatorname{End}\left(N^{*}(X)\right) .
$$

Here Sur is the Surjection operad of McClure-Smith [MS03, and Berger-Fresse [BF04, the first arrow is the Table Reduction morphism of [BF04], and the second is the natural Sur-algebra structure introduced in MS03 and BF04.

4.1. The Surjection operad. We review the definition of the Surjection operad of McClure-Smith MS03. and Berger-Fresse [BF04.

For a non-negative integer $n$ define

$$
\bar{n}=\left\{\begin{array}{cc}
\{1, \ldots, n\} & n>0 \\
\emptyset & n=0 .
\end{array}\right.
$$

Fix $r \geq 1$ and consider all $n \geq 0$. We make the free $\mathbb{F}_{2}$-module generated by all functions $s: \bar{n} \rightarrow \bar{r}$ into a chain complex by declaring the degree of $s: \bar{n} \rightarrow \bar{r}$ to be $r-n$ and its boundary to be

$$
\partial s=\sum_{k=1}^{n} s \circ \delta_{k}
$$

where $\delta_{k}: \overline{n-1} \rightarrow \bar{n}$ is the injective order preserving function missing $k$.

We define $\operatorname{Sur}(r)$ to be the quotient of this chain complex by the submodule generated by the functions $s: \bar{n} \rightarrow \bar{r}$ which are either non-surjective or for which $s(i)$ equals $s(i+1)$ for some $i$.

The chain complex $\operatorname{Sur}(r)$ carries an action of $\Sigma_{r}=\{\bar{r} \rightarrow \bar{r} \mid$ bijective $\}$ given by composition. The collection $\operatorname{Sur}=\{\operatorname{Sur}(r)\}_{r \geq 0}$ is an operad with partial composition $\circ_{p}: \operatorname{Sur}\left(r^{\prime}\right) \otimes \operatorname{Sur}(r) \rightarrow \operatorname{Sur}\left(r+r^{\prime}-1\right)$ defined on two generators $s: \bar{n} \rightarrow \bar{r}$ and $s^{\prime}: \overline{n^{\prime}} \rightarrow \overline{r^{\prime}}$ as follows. Represent the surjections $s$ and $s^{\prime}$ by sequences $(s(1), \ldots, s(n))$ and $\left(s^{\prime}(1), \ldots, s^{\prime}\left(n^{\prime}\right)\right)$ and suppose that $p$ appears $k$ times in the sequence representing $s^{\prime}$ as $p=s^{\prime}\left(i_{1}\right)=\cdots=s^{\prime}\left(i_{k}\right)$. Denote the set of all tuples

$$
1=j_{0} \leq j_{1} \leq \cdots \leq j_{k}=n
$$

by $J(k, n)$ and for each such tuple consider the subsequences

$$
\left(s\left(j_{0}\right), \ldots, s\left(j_{1}\right)\right) \quad\left(s\left(j_{1}\right), \ldots, s\left(j_{2}\right)\right) \quad \ldots \quad\left(s\left(j_{k-1}\right), \ldots, s\left(j_{k}\right)\right) .
$$

Then, in $\left(s^{\prime}(1), \ldots, s^{\prime}(n)\right)$, replace the value $s^{\prime}\left(i_{t}\right)$ by $\left(s\left(j_{t-1}\right), \ldots, s\left(j_{t}\right)\right)$. In addition, increase the terms $s(j)$ by $r-1$ and the terms $s^{\prime}(i)$ such that $s^{\prime}(i)>r$ by $m-1$. The surjection $s^{\prime} \circ_{p} s$ is represented by the sum, parametrized by $J(k, n)$, of these resulting sequences. 
Example 15. Let us consider

$$
s=(1,2,1) \quad s^{\prime}=(1,2,3,2,1) .
$$

Then, $s^{\prime} \circ_{2} s$ equals

$$
(1,(2,3,2), 4,(2), 1)+(1,(2,3), 4,(3,2), 1)+(1,(2), 4,(2,3,2), 1)
$$

where the internal parenthesis are included solely for expository purposes.

4.2. The Table Reduction morphism. We review the definition of the Table Reduction operad morphism

$$
T R: \mathcal{E} \rightarrow \text { Sur }
$$

introduced in [BF04].

Given a basis element $\left(\sigma_{0}, \ldots, \sigma_{n}\right) \in \mathcal{E}(r)_{n}$ we define

$$
T R\left(\sigma_{0}, \ldots, \sigma_{n}\right)=\sum_{a} s_{a}
$$

as a sum of surjections

$$
s_{a}:\{1, \ldots, n+r\} \rightarrow\{1, \ldots, r\}
$$

parametrized by all $a=\left(a_{0}, \ldots, a_{n}\right)$ with each $a_{i} \geq 1$ and $a_{0}+\cdots+a_{n}=n+r$.

For one such tuple $a$ we now describe its associated surjection $s_{a}$. Consider the table

$$
\mid \begin{array}{ccc}
\sigma_{0}(1) & \cdots & \sigma_{0}(r) \\
\sigma_{1}(1) & \cdots & \sigma_{1}(r) \\
\vdots & \ddots & \vdots \\
\sigma_{n}(1) & \cdots & \sigma_{n}(r)
\end{array}
$$

define recursively

$$
A_{-1}=0 \quad A_{i}=A_{i-1}+a_{i .}
$$

For $k \in\{1, \ldots, n+r\}$ we identify $i \in\{1, \ldots, n\}$ such that $A_{i-1}<k \leq A_{i}$ and define $s_{a}(k)$ to be the $\left(k-A_{i-1}\right)$-th element in $\left(\sigma_{i}(1), \ldots, \sigma_{i}(r)\right)$ not in

$$
\left\{s_{a}(j) \mid j<k \text { and } j \neq A_{0}, \ldots, A_{i-1}\right\} .
$$

Example 16. Let us compute $T R((23), e,(12)(34))$. Its associated table is

$$
\begin{array}{|llll}
1 & 3 & 2 & 4 \\
1 & 2 & 3 & 4 \\
2 & 1 & 4 & 3
\end{array}
$$

and the tuples parametrizing the sum correspond to all permutations of $\{(1,1,4),(1,2,3),(2,2,2)\}$. Then,

$$
\begin{aligned}
\operatorname{TR}((23), e,(12)(34)) & =(1,3,2,3,4,3) \\
& +(1,1,2,3,4,4)+(1,3,2,4,4,4)+(1,1,2,1,4,3) \\
& +(1,3,2,2,2,4)+(1,1,2,3,4,3)+(1,3,2,2,4,4) \\
& +(1,3,2,3,4,4)+(1,1,2,2,4,3)+(1,3,2,2,4,3) \\
& =(1,3,2,3,4,3) .
\end{aligned}
$$


4.3. The diagonal and join maps. We review the natural $S u r$-algebra structure on $N^{*}(X)$ introduced in MS03 and BF04] using the perspective presented in [MM18b]. We notice that this Sur-algebra structure provides $N^{*}(X)$ with an $\mathcal{E}$-algebra structure

$$
\mathcal{E} \rightarrow \operatorname{Surj} \rightarrow \operatorname{End}\left(N^{*}(X)\right) .
$$

Let $\boldsymbol{\Delta}^{n}$ be the representable simplicial set $\operatorname{Hom}_{\boldsymbol{\Delta}}(-,[n])$. We identify a morphism $[m] \rightarrow[n]$ with its image $\left\{v_{0}, \ldots, v_{m}\right\} \subseteq\{0, \ldots, n\}$. As usual, it suffices to describe the $S u r j$-structure for representable simplicial sets only.

Let $\Delta: N^{*}\left(\boldsymbol{\Delta}^{n}\right) \rightarrow N^{*}\left(\boldsymbol{\Delta}^{n}\right)^{\otimes 2}$ be defined on basis elements by

$$
\Delta\left\{v_{0}, \ldots, v_{m}\right\}=\sum_{i=0}^{m}\left\{v_{0}, \ldots, v_{i}\right\} \otimes\left\{v_{i}, \ldots, v_{m}\right\}
$$

and, for any $k \geq 2$, let $*: N^{*}\left(\boldsymbol{\Delta}^{n}\right)^{\otimes k} \rightarrow N^{*}\left(\boldsymbol{\Delta}^{n}\right)$ be defined on basis elements by

$$
*\left(a_{1} \otimes \cdots \otimes a_{k}\right)= \begin{cases}\bigcup_{i=1}^{k} a_{i} & \forall i<j, a_{i} \cap a_{j}=\emptyset \\ 0 & \exists i<j, a_{i} \cap a_{j} \neq \emptyset .\end{cases}
$$

We notice that $\Delta$ is equal to the composition of the Alexander-Whitney map and the doubling map

$$
\begin{aligned}
N^{*}\left(\boldsymbol{\Delta}^{n}\right) & \rightarrow N^{*}\left(\boldsymbol{\Delta}^{n} \times \boldsymbol{\Delta}^{n}\right) \\
a & \mapsto a \times a
\end{aligned}
$$

and that $*$ is commutative.

We now describe the Sur-algebra structure on $N^{*}\left(\boldsymbol{\Delta}^{n}\right)$. Let

$$
s:\{1, \ldots, n+r\} \rightarrow\{1, \ldots, r\}
$$

be a surjection, then $s\left(\alpha_{1} \otimes \cdots \otimes \alpha_{r}\right)$ is defined by

$$
s\left(\alpha_{1} \otimes \cdots \otimes \alpha_{r}\right)(a)=\left(\alpha_{1} \otimes \cdots \otimes \alpha_{r}\right)\left(*_{s^{-1}(1)} \otimes \cdots \otimes *_{s^{-1}(r)}\right) \Delta^{r+d-1}(a)
$$

where $\Delta^{k}$ is recursively defined by

$$
\begin{aligned}
& \Delta^{1}=\Delta \\
& \Delta^{k+1}=\left(\Delta \otimes \mathrm{id}^{\otimes k}\right) \Delta^{k}
\end{aligned}
$$

and $*_{s^{-1}(i)}$ is given by applying $*$ to the factors in positions $s^{-1}(i)$.

Remark 17. This $S u r j$-algebra structure on normalized cochains of simplicial sets is induced, as explained in [MM18c, from an explicit cellular $E_{\infty}$-bialgebra structure on the geometric realization of $\boldsymbol{\Delta}^{\mathbf{1}}$.

\section{Appendix A. Explicit examples}

Let $\boldsymbol{\Delta}_{n}$ be the $n$-th representable simplicial set and denote $\{0, \ldots, n\} \in N_{n}\left(\boldsymbol{\Delta}^{n}\right)$ by $\mathrm{id}_{n}$. In this appendix, we will compute for an arbitrary pair of homogeneous cocycles $\alpha, \beta \in N^{*}\left(\boldsymbol{\Delta}_{n}\right)$ the value

$$
\zeta_{i}(\alpha \otimes \beta)\left(\operatorname{id}_{n}\right)
$$

in terms of $\alpha$ and $\beta$. We will restrict to $i=0,1,2$ and $n$ the smallest integer for which this value is not identically 0 .

Recall that

$$
\tilde{x}_{i}=\left(e,(12), e, \ldots,(12)^{i}\right) \in \mathcal{E}(2)
$$

and that our Cartan $i$-coboundary is defined by

$$
\zeta_{i}(\alpha \otimes \beta)=T R\left(H_{1}+H_{2}\right)\left(\tilde{x}_{i}\right)(\alpha \otimes \alpha \otimes \beta \otimes \beta)
$$


where $T R: \mathcal{E} \rightarrow \operatorname{Surj}$ is the Table Reduction morphism and

$$
\begin{gathered}
H_{1}\left(\sigma_{0}, \ldots, \sigma_{n}\right)=\sum_{i=0}^{n}\left((23) f \sigma_{0}, \ldots,(23) f \sigma_{i}, g \sigma_{i}, \ldots, g \sigma_{n}\right) \\
H_{2}\left(\sigma_{0}, \ldots, \sigma_{n}\right)=N_{*}\left(\circ_{E}\right)\left((e, \ldots, e) \otimes S H I\left(\sigma_{0}, \ldots, \sigma_{n}\right)^{\otimes 2}\right) .
\end{gathered}
$$

We remark that the cochain $\zeta_{i}(\alpha \otimes \beta)$ witnesses the relation

$$
S q^{p+q-i}([\alpha][\beta])=\sum_{p+q-i=j+k} S q^{j}([\alpha]) S q^{k}([\beta])
$$

where $-p$ and $-q$ are the degrees of $\alpha$ and $\beta$.

Example 18. For $i=0$, we have

$$
H_{1}\left(\tilde{x}_{0}\right)=((23) f e, g e)=((23), e)
$$

and

Therefore,

$$
H_{2}\left(\tilde{x}_{0}\right)=0 \text {. }
$$

$$
\operatorname{TR}\left(\left(H_{1}+H_{2}\right)\left(\tilde{x}_{0}\right)\right)=(1,3,2,3,4)
$$

and

$$
\zeta_{0}(\alpha \otimes \beta)\{0,1,2,3\}=\alpha\{0,1\} \alpha\{1,2\} \beta\{1,2\} \beta\{2,3\} .
$$

Example 19. For $i=1$, we have

$$
H_{1}\left(\tilde{x}_{1}\right)=((23), e,(12)(34))+((23),(23)(13)(24),(12)(34))
$$

and

Therefore,

$$
H_{2}\left(\tilde{x}_{1}\right)=(e,(34),(12)(34)) .
$$

$$
T R\left(\left(H_{1}+H_{2}\right)\left(\tilde{x}_{1}\right)\right)=(1,3,2,3,4,3)+(1,2,4,1,4,3)
$$

and

$$
\begin{aligned}
\zeta_{1}(\alpha \otimes \beta)\{0,1,2,3,4\} & =\alpha\{0,1\} \alpha\{1,2\} \beta\{1,2,4\} \beta\{2,3,4\} \\
& +\alpha\{0,2,3\} \alpha\{0,1,2\} \beta\{3,4\} \beta\{2,3\} .
\end{aligned}
$$

Example 20. For $i=2$, we have

$$
\begin{aligned}
H_{1}\left(\tilde{x}_{2}\right) & =((23), e,(12)(34), e)+((23),(12)(34)(23),(12)(34), e) \\
& +((23),(12)(34)(23),(23), e)
\end{aligned}
$$

and

$$
\begin{aligned}
H_{2}\left(\tilde{x}_{2}\right) & =(e,(34),(12)(34),(12))+(e, e,(12), e) \\
& +(e,(34), e,(12))+(e,(12)(34),(12), e) .
\end{aligned}
$$

Therefore,

$$
\begin{aligned}
\operatorname{TR}\left(\left(H_{1}+H_{2}\right)\left(\tilde{x}_{2}\right)\right) & =(1,3,2,3,4,3,4)+(1,2,4,1,4,3,4) \\
& +(1,2,1,3,2,3,4)+(1,3,2,3,2,3,4)
\end{aligned}
$$

and

$$
\begin{aligned}
\zeta_{2}(\alpha \otimes \beta)\{0,1,2,3,4,5\} & =\alpha\{0,1\} \alpha\{1,2\} \beta\{1,2,3,4\} \beta\{2,3,4,5\} \\
& +\alpha\{0,1\} \alpha\{1,2\} \beta\{1,2,4,5\} \beta\{2,3,4,5\} \\
& +\alpha\{0,2,3\} \alpha\{0,1,2\} \beta\{3,4,5\} \beta\{2,3,5\} \\
& +\alpha\{0,1,2,3\} \alpha\{0,1,3,4\} \beta\{3,4\} \beta\{4,5\} \\
& +\alpha\{0,1,2,3\} \alpha\{1,2,3,4\} \beta\{3,4\} \beta\{4,5\} .
\end{aligned}
$$




\section{REFERENCES}

[BF04] Clemens Berger and Benoit Fresse. Combinatorial operad actions on cochains. In Mathematical Proceedings of the Cambridge Philosophical Society, volume 137, pages 135-174. Cambridge University Press, 2004.

[BM16] Greg Brumfiel and John Morgan. The pontrjagin dual of 3 -dimensional spin bordism. arXiv preprint arXiv:1612.02860, 2016.

[BM18] Greg Brumfiel and John Morgan. Quadratic functions of cocycles and pin structures. arXiv preprint arXiv:1808.10484, 2018.

[EML53] Samuel Eilenberg and Saunders Mac Lane. On the groups $H(\pi, n)$, I. Ann. of Math.(2), 58(1):55-106, 1953.

[KT17] Anton Kapustin and Ryan Thorngren. Fermionic spt phases in higher dimensions and bosonization. Journal of High Energy Physics, 2017(10):80, 2017.

[LV12] Jean-Louis Loday and Bruno Vallette. Algebraic operads, volume 346 of Grundlehren der Mathematischen Wissenschaften [Fundamental Principles of Mathematical Sciences]. Springer, Heidelberg, 2012.

[May70] J Peter May. A general algebraic approach to Steenrod operations. In The Steenrod Algebra and its Applications: a conference to celebrate NE Steenrod's sixtieth birthday, pages 153-231. Springer, 1970.

[MM18a] Anibal M. Medina-Mardones. An axiomatic characterization of Steenrod's cup- $i$ products. arXiv preprint arXiv:1810.06505, 2018.

[MM18b] Anibal. M. Medina-Mardones. A finitely presented $E_{\infty}$-prop I: algebraic context. arXiv preprint arXiv:1808.00854, 2018.

[MM18c] Anibal M. Medina-Mardones. A finitely presented $E_{\infty}$-prop II: cellular context. arXiv preprint arXiv:1808.07132, 2018.

[MM18d] Anibal M. Medina-Mardones. Persistence Steenrod modules. arXiv preprint arXiv:1812.05031, 2018.

[MM19] Anibal M. Medina-Mardones. An algebraic representation of globular sets. arXiv preprint arXiv:1906.01011, 2019.

[MS03] James McClure and Jeffrey Smith. Multivariable cochain operations and little n-cubes. Journal of the American Mathematical Society, 16(3):681-704, 2003.

[Rea00] Pedro Real. Homological perturbation theory and associativity. Homology, Homotopy and Applications, 2(1):51-88, 2000.

[Rub91] J Rubio. Homologie effective des espaces de lacets itérés: un logiciel. These de doctorat de lInstitut Fourier, Grenoble, 1991.

[SE62] Norman Earl Steenrod and David BA Epstein. Cohomology operations. Number 50-51. Princeton University Press, 1962.

[Ste47] Norman E Steenrod. Products of cocycles and extensions of mappings. Annals of Mathematics, pages 290-320, 1947.

E-mail address: anibal.medinamardones@epfl.ch

Laboratory for Topology and Neuroscience, cole Polytechnique Fdrale de Lausanne, Switzerland

Department of Mathematics, University of Notre Dame du Lac, Notre Dame, in, USA 